Meta

Journal des traducteurs

Translators' Journal

\title{
L'exil comme métaphore de la traduction : la pure langue de Walter Benjamin et la langue vive de Carles Riba
}

\section{Enrique Pérez Cristóbal}

Volume 56, numéro 4, décembre 2011

URI : https://id.erudit.org/iderudit/1011256ar

DOI : https://doi.org/10.7202/1011256ar

Aller au sommaire du numéro

\section{Éditeur(s)}

Les Presses de l’Université de Montréal

ISSN

0026-0452 (imprimé)

1492-1421 (numérique)

Découvrir la revue

Citer cet article

Pérez Cristóbal, E. (2011). L’exil comme métaphore de la traduction : la pure langue de Walter Benjamin et la langue vive de Carles Riba. Meta, 56(4), 852-860. https://doi.org/10.7202/1011256ar

\section{Résumé de l'article}

L'exil a été à maintes reprises lu comme une possible métaphore de la traduction, ou en tout cas comme une expérience structuralement proche de celle de la traduction. Walter Benjamin (1892-1940) et Carles Riba (1893-1959), tout en partant de la lecture de Hölderlin, et sans jamais arriver à se lire l'un l'autre, ont élaboré à la même époque une poétique de la traduction à la fois commune et antagonique. Benjamin, quant à lui, a spéculé sur la possibilité d'une " pure langue " exilée, comme on sait, à l'intérieur des différentes langues empiriques, tandis que Riba a travaillé le catalan noucentista - en tant que poète et traducteur - presque comme cette langue hypothétique supposée par Benjamin. L’objet du présent article est de mesurer la distance existant entre la pure langue (reine Sprache) de Benjamin et la langue vive (llengua viva) de Riba, toujours à partir de l'expérience de l'exil comme possible modèle anthropologique et structural de la traduction. 


\title{
L'exil comme métaphore de la traduction: la pure langue de Walter Benjamin et la langue vive de Carles Riba
}

\author{
ENRIQUE PÉREZ CRISTÓBAL \\ Université d'Evry Val-d'Essonne, Evry, France \\ enriqueolallaes@gmail.com
}

\begin{abstract}
RÉSUMÉ
L'exil a été à maintes reprises lu comme une possible métaphore de la traduction, ou en tout cas comme une expérience structuralement proche de celle de la traduction. Walter Benjamin (1892-1940) et Carles Riba (1893-1959), tout en partant de la lecture de Hölderlin, et sans jamais arriver à se lire l'un l'autre, ont élaboré à la même époque une poétique de la traduction à la fois commune et antagonique. Benjamin, quant à lui, a spéculé sur la possibilité d'une «pure langue» exilée, comme on sait, à l'intérieur des différentes langues empiriques, tandis que Riba a travaillé le catalan noucentista - en tant que poète et traducteur - presque comme cette langue hypothétique supposée par Benjamin. L'objet du présent article est de mesurer la distance existant entre la pure langue (reine Sprache) de Benjamin et la langue vive (llengua viva) de Riba, toujours à partir de l'expérience de l'exil comme possible modèle anthropologique et structural de la traduction.
\end{abstract}

\footnotetext{
ABSTRACT as an anthropological and structural model of translation.

\section{MOTS-CLÉS/KEYWORDS}

exil, Benjamin, Riba, Hölderlin, pure langue, langue vive

exile, Benjamin, Riba, Hölderlin, pure language, live language
}

The exile was several times read like a possible metaphor of translation, or at least like an experience structurally analogous to translation. Walter Benjamin (1892-1940) and Carles Riba (1893-1959), from the reading of Hölderlin, though they never suspected each other's thoughts, worked both on a poetic of translation at once common and antagonistic. Benjamin speculated on the possibility of a "pure language" in exile and fractured inside the empirical languages, while Riba worked the noucentista Catalan - as poet and translator - almost like this hypothetical language supposed by Benjamin. The purpose of this article is to measure the common distance between the Benjamin's pure language (reine Sprache) and Riba's live language (llengua viva), always from the experience of exile

Le 28 janvier 1939, Yeats mourait dans le sud de la France. Ce même jour, un autre poète d'ascendance symboliste, Antonio Machado, traversait la frontière francoespagnole. Il commençait son exil. Néanmoins, il mourra quelques jours après, aussi dans le sud de la France. Il faut se rappeler que la France fut la terre par excellence du Symbolisme pour comprendre tout le sens d'une des phrases le plus souvent citées d'Antonio Machado, au début de son exil: «¡Hallarme en Francia, y como un mendigo $!^{1} »$ (Me trouver en France, comme un mendiant!). Paroles prononcées - ce même 28 janvier 1939 - à l'adresse de Carles Riba, peut-être le dernier grand poète symboliste européen. 
Paroles que dans un exil inverse, presque un an après, pourrait bien avoir prononcées à Portbou Walter Benjamin - «wie ein Bettler, im Spanien, ich mich zu sehen" (Me trouver comme un mendiant en Espagne; traduction de l'auteur) -, quelques heures avant de mettre un terme à sa vie. Paroles non dites, non écoutées du moins, mais qui nous aident à mieux comprendre celles de Machado en France. Elles nous rappellent, à leur tour, que l'Espagne fut un temps le berceau de la première école de traducteurs en Europe, en même temps que le foyer de la Cabale. Ce qui aurait été impensable avant l'exil des premiers cabalistes provençaux, qui traversèrent les Pyrénées, comme plus tard le feraient Walter Benjamin et Carles Riba.

Entre ces deux exils s'écrit toute une page de l'histoire de l'Europe, incompréhensible en dehors du rapport - imposé par cette histoire même - entre traduction et exil. Relation essentielle pour arriver à comprendre la conception linguistique qui soutient Symbolisme et Cabale, dominés tous deux par une croyance en un absolu linguistique au-delà de la simple communication (Scholem 1970/2006: 100). Peut-être un des plus grands paradoxes qui a nourri et bloqué, presque dans la même mesure, l'évolution de la pensée linguistique. La foi en quelque chose d'inexprimable qui habite en elles depuis la nuit des temps, moins capable de refléter les choses que d'exprimer leurs rapports (Rancière 1998: 43). Presque exilée aujourd'hui, cette foi en notre horizon linguistique n'a cessé pourtant d'alimenter la pensée de certains auteurs modernes, parmi lesquels Carles Riba et Walter Benjamin.

Une foi à laquelle Benjamin aboutira à travers une singulière métaphysique du langage, née d'une conception de la traduction en tant que rédemption (Erlösung) d'une langue exilée (gebannt), que les langues empiriques à la fois contiendraient et recouvriraient, désigneraient et abâtardiraient. Cette langue est pour Benjamin - à mi-chemin de la langue adamique de la Genèse et de la raison pure de Kant - la semence voilée d'une langue plus haute, une pure langue (reine Sprache) (Benjamin 1972: 19). Une langue que Riba, pour sa part, et grâce à l'expérience commune de la traduction et de l'exil, comprendrait - en reprenant la théorie de la parole vive (paraula viva) de Joan Maragall - sous la forme de langue vive (llengua viva) (Riba 1988: 81, 163).

Néanmoins, ce traducteur de l'antiquité, que fut Riba, et cet archéologue de la modernité, qu'on reconnaît aujourd'hui en Benjamin, peuvent sembler presque antagoniques en tant qu'hommes de lettres et traducteurs. "Outsider" sa vie durant, Benjamin a toujours travaillé en dehors du milieu académique, essayant de devenir en France le premier critique de la littérature allemande. Un prosateur comme il y en a peu, installé à la fois à l'intérieur de l'immanence du poème (das Gedichtete) et hors de son expression la plus habituelle. Quant à Riba, on peut en dire presque tout le contraire: bientôt devenu à la fois poète et philologue - à travers ses traductions des Bucoliques de Virgile (-39 av. J.-C./1911) et de l'Odyssée d'Homère (viri s. av. J.-C./1919) ${ }^{3}$-, il travaillera comme professeur de littératures classiques à la Mancomunitat de Catalunya et, une fois créée la Fundació Bernard Metge, deviendra finalement agrégé de grec.

Meilleur traducteur que théoricien de la traduction, à l'inverse de Benjamin, Riba tire de son expérience de la traduction la plupart de ses conclusions sur la tâche du traducteur, tandis que chez Benjamin il y a un abîme entre son expérience comme traducteur - notamment d'auteurs modernes français - et sa philosophie de la traduction. Les différents paradoxes que pose pour chacun d'eux la traduction - même 
à partir du couple fidélité-liberté - semblent être un motif de plus pour les éloigner: la traduction comme restitution (Wiedergabe) ou comme reproduction, comme révélation (Offenbarung) ou occultation, comme écho (Widerhall) ou reflet, pour Benjamin; comme re-création (recreació) ou transport, comme renaissance (renaixement) ou survivance, comme révolte (rebellió) ou soumission, chez Riba. Si celui-ci, à titre de disciple de Karl Vossler, a parfois recours au vocabulaire linguistique afin de mieux saisir la distance entre la littéralité et le sens que pose toute traduction, la pensée de Benjamin restera toujours étrangère à la linguistique, plus proche de la métaphysique langagière d'Hamann que de la stylistique de Spitzer.

Malgré toutes leurs différences, et sans jamais arriver à se connaître ${ }^{4}$, on peut dire d'une certaine façon que Riba avait commencé à aborder dans la pratique certains problèmes sur lesquels Benjamin ne cessait de théoriser. La pure langue de Benjamin - toujours tue mais encore écoutable - était pour Riba le catalan autour de 1900: une vieille langue mais à la fois trop jeune, très soutenue et en même temps primitive; une langue encore en état de façonnage (estat de plasmació, Riba 1985: 164), dont la potentialité indéfinie lui permettait en même temps d'aboutir à de nouvelles formes et de les décristalliser. Une langue perdue et pourtant une langue-quivient, ni archaïque ni actuelle, mais une langue qu'on parlera très certainement (Riba 1988: 164; Berman 2008: 115).

Dans la préhistoire de ces deux langues - pure pour Benjamin, d'après la terminologie kantienne, vive pour Riba, en suivant Maragall, dans les deux cas très près du Romantisme allemand -, l'hébreu jouera un rôle non négligeable. Une préhistoire liée en quelque sorte à la critique de la traduction, particulièrement de deux textes bibliques: la traduction au catalan de la Genèse, faite en 1915 par Frederic Casclar, et du Cantique des Cantiques vers l'allemand, accomplie en 1917 par Gershom Scholem. Malgré son ignorance non seulement de l'hébreu mais aussi du texte de Salomon, Benjamin - qui vient de lire les traductions de Pindare faites par Hölderlin - condamnera sans ambages la traduction de Scholem, puisqu'elle n'arrive pas à faire passer l'hébreu et l'allemand dans une sphère unique (in eine Sphäre eingehen, Benjamin 1993: 142). Quant à Riba, qui traduira lui même trois ans plus tard le Cantique des Cantiques et le Livre de Ruth, il remarque dans la version de Clascar sa capacité justement de donner aux deux langues une vie parallèle (paral.lela vida, Riba 1985: 163); fait possible, selon Riba, grâce tant à l'état de façonnage et décristallisation du catalan noucentista, que de la nature non exacte et vivante de l'hébreu:

[1]'hebreu no és una llengua exacta [...]. En canvi, cada mot té una fesomia que mostra el seu llinatge en un relleu vivent [...]. (Riba 1985: 163)

[l]'hébreu n'est pas une langue exacte [...]. En revanche, chaque mot présente une physionomie qui montre son lien avec un relief vivant [...]. (Traduction de l'auteur)

Ajoutons que cette «sphère unique» - déduite par Benjamin en réfléchissant sur les traductions historiques de Hölderlin - est déjà très proche de l'idéal de traduction proposée dans Die Aufgabe des Übersetzers, c'est-à-dire, la version interlinéaire du texte sacré ( Die Interlinearversion des Heiligen Textes ist das Urbild oder Ideal aller Übersetzung", Benjamin 1972: 21). Une sphère unique comprise, dans la préface à sa traduction des Tableaux parisiens de Baudelaire, en tant que co-relation vivante (Zusammenhang des Lebens, Benjamin 1972: 10). Et pour cause, puisque Benjamin va penser le rapport de la traduction et de l'œuvre dans la dimension de la vie - non 
loin de cette "paral-lela vida» soutenue par Riba -, conçue comme nature et à la fois comme histoire, comme intimité et expérience (Berman 2008: 70). Une vie qui cache et révèle en même temps le mouvement langagier (Sprachbewegung), étant donné que la tangente de ce mouvement - dans le processus de traduction, selon Benjamin touche à peine un instant le cercle du sens, pour suivre encore sa trajectoire (Benjamin 1972: 20). Voilà une nouvelle raison, pour Benjamin, de l'exil de la pure langue dans les langues empiriques.

On peut affirmer ainsi du catalan de 1900 ce que Benjamin affirmait de la finalité de la traduction, dans une des phrases centrales de Die Aufgabe des Übersetzers:

[...] das Symbolisierende zum Symbolisierten selbst zu machen, die reine Sprache gestaltet der Sprachbewegung zurückzugewinnen. (Benjamin 1972: 19)

[...] faire du symbolisant le symbolisé, retrouver la pure langue structurée dans le mouvement langagier. (Traduction d'Antoine Berman [Berman 2008: 176])

Mouvement dans lequel Riba va reconnaître non seulement l'objet de la traduction ${ }^{5}$, mais encore la procédure à suivre pour pouvoir traduire des textes tels que les tragédies d'Eschyle ou l'Odyssée:

Llavors el procediment és obvi: lliurar-se sense reserves al moviment poètic, seguint-ne els meandres amb obstinada atenció, revivint cada intuïció amb una alta humilitat; tot això, en funció de la llengua a la qual tradueix, però no sotmeten-se a l'estil que imposi ella, sinò creant en ella un estil. (Riba 1953: 11)

La procédure à suivre est évidente: s'abandonner sans réserve au mouvement poétique, en suivant ses méandres avec une attention obstinée, revivant chaque intuition avec une grande humilité; tout cela en fonction de la langue que l'on traduit, sans se soumettre au style qu'elle impose, mais en créant un style propre en elle-même. (Traduction de l'auteur)

Ce mouvement, dans les deux cas, il faut le dire, est un mouvement qui lie la réflexion traductologique à l'expérience d'un certain exil, vécu par Benjamin presque métaphysiquement, religieusement par Riba. Tous deux - depuis la guerre civile espagnole pour le Catalan, après avoir connu de multiples faux pas dans sa vie professionnelle pour ce qui est de Benjamin - ont confié à la langue une grande partie de leurs espérances. Mais tandis que Riba a lié ses espoirs à la patrie espérée (pàtria expectant; Riba 1984: 236), Benjamin n'a jamais eu une expérience du pays natal (Heimat) semblable, ni avec la langue allemande - comme c'est le cas chez Heidegger - ni finalement avec l'hébreu, à la façon problématique de Scholem (1970/2006). C'est peut-être pour cela que Riba s'interroge à plusieurs reprises sur le mythe classique d'Ulysse, tandis que Benjamin se tourne vers le mythe moderne de Joseph K. Deux formes bien distinctes de se sentir ailleurs, en apparence antagoniques, de la même façon que la pensée grecque peut apparaître parfois comme antagonique vis-à-vis de la pensée juive, alors même qu'elles proviennent pourtant d'une même source. Comme le pense Benjamin, à propos de Kafka (ein anderer Odysseus [un autre Ulysse]) :

[u]nter den Ahnen, die Kafka in der Antike hat, den jüdischen und den chinesischen [...], ist dieser griechische nicht zu vergessen. (Benjamin 1977: 415)

[p]armi les ancêtres antiques de Kafka, les Juifs et les Chinois [...], cet ancêtre grec ne doit pas être oublié. (Traduction de l'auteur) 
C'est peut-être pour cela que Riba - un des plus grands poètes-traducteurs modernes du grec antique - offrira à son tour la première traduction ibérique de Kafka, publiée en décembre 1924, dans la revue La Mà trencada ${ }^{6}$.

Les deux traductions ribiennes de l'Odyssée $(1919 ; 1948)^{7}$, ainsi que ses propres poèmes «homériques» $(1919 ; 1942)^{8}$, montrent bien l'impact de l'exil sur cette expérience de sortie et de rentrée qui est aussi celle de la traduction. Si dans sa jeunesse Riba éprouve surtout le mouvement de sortie du mythe d'Ulysse ${ }^{9}$ - ce qu'on peut constater dans sa première traduction de l'Odyssée -, pendant et après son exil (19391943), ce sera le mouvement de rentrée, aussi bien vers la pàtria expectant que vers sa langue. Un élan qui dominera non seulement sa poésie, mais aussi la plupart de ses traductions, notamment sa deuxième traduction de l'Odyssée et ses versions de Hölderlin (Malé 2006: 22-26). Quand Riba essaie de traduire à nouveau le poème d'Ulysse, presque trente ans après sa première traduction, il le ferait moins en tant que philologue expérimenté que comme poète et exilé. Un exil physique que Riba n'hésitera pas à mettre en rapport avec un certain exil linguistique:

[...] Entre nosaltres humans,

déus! els mots són només per a entendre'ns i no per a entendre'ls: són el commençament, just un senyal del sentit.

Semblen precedir-nos camí del misteri i ens deixen foscos davant d'un brocat, tristos a un eco que fuig.

Cal la secreta clau: un record que ve de vosaltres, déus! i que no ens ateny fins que ja hem arribat.

(Riba 1984: 225) ${ }^{10}$

\section{[...] Parmi nous, humains,}

dieux!, les paroles sont juste pour nous comprendre, pas pour les comprendre: elles sont le commencement, à peine un signal du sens.

Elles semblent nous précéder le mystère, et nous rendent obscurs face à un brocart, tristes devant un écho qui fuit.

Nous avons besoin de la clé secrète: un souvenir qui provient de vous, dieux!, et qui ne nous atteint que quand on est arrivé.

(Traduction de l'auteur)

Voués à un usage presque entièrement communicatif du langage, exilés de ce chemin de la trascendence, à peine nous reste-t-il de lui qu'un signal. Un écho dont le souvenir, selon Riba, nous parvient seulement à la fin de notre vie. Les paroles, avant ce moment, et face à ce chemin, nous offrent seulement ou bien une possibilité de communication ou bien l'artifice d'un discours, aussi beau qu'un brocart, mais insuffisant.

Une fois de retour en Catalogne en 1943 - où l'expression publique du catalan est devenue interdite -, Riba mettra en pratique cette expérience de la parole en exil dans sa traduction de l'Odyssée. Un travail d'une telle envergure qu'un critique de l'époque affirmera sans hésitation qu'avec l'Odissea de Riba et le Nabí de Carner on pourrait reconstruire la langue catalane, si celle-ci arrivait à disparaître (Medina 1987: 16).

On peut dire qu'avant son exil Riba pense la traduction notamment comme une manière de rendre service à un autre, en même temps que comme une possibilité de s'approprier le destin de cet autre: 
Traduir és, en certa arièlica manera, també servir: no pot anar sensa aquella humilitat [...] de qui es fa seu un destí d'altri. (Riba 1986: 83)

Traduire c'est, d'une façon ariélienne, à la fois servir: elle ne va pas sans l'humilité [...] de celui-là qui fait sien le destin d'un autre. (Traduction de l'auteur)

Une fois Riba exilé, la traduction deviendra pour lui non seulement un moyen de faire sien un destin d'un autre, mais surtout un moyen de faire passer sa propre voix - transformée déjà par l'exil - à travers la voix d'un autre («fer passar la seva pròpia veu per la d'un altre », Riba 1988: 188). Et pour cause, puisque cette voix sera non seulement celle des poètes homériques, mais la voix de Hölderlin - que Riba avait commencé à traduire déjà en 1920 (Medina 1987 : 10). Encore que, selon Ferrater, ces premières traductions étaient à peine une préparation aux Elegies de Bierville (titre en catalan), c'est-à-dire l'exercice d'écriture et de traduction de son propre exil:

Tal com ho era, des de la banda oposada, l'aprofundiment en el grec. Però un dia es va a produir la confluència, en forma de les Elegies de Bierville: el senyal més fort, i ja indeleble, de la petja de Hölderlin damunt de la poesia catalana. (Ferrater 1979: 39) De la même façon que l'était, à l'inverse, l'approfondissement du grec. Mais un jour aura lieu la confluence, sous la forme des Élégies de Bierville: le signe plus fort, et désormais indélébile, de la trace de Hölderlin sur la poésie catalane. (Traduction de l'auteur)

Confluence, comme si c'était littéralement un fleuve, avec le poète et traducteur qui peut-être a le plus profondément réfléchi sur la co-relation vivante entre le national et l'étranger (Berman 1984/2007: 250-278) ${ }^{11}$. Aussi bien pour Riba que pour Benjamin, c'est la lecture du poète de Heimkunft (Hölderlin 1801/2003) ${ }^{12}$ qui constituera une des expériences fondamentales leur permettant de penser dans toute sa complexité et sa richesse la co-relation entre la langue et l'exil, l'oralité et le dialecte, la traduction et cette langue pure et vive. «Depuis des années, écrit Benjamin en 1916, la lumière qui pour moi rayonne dans cette nuit est celle de Hölderlin » (Benjamin 1979: 122). Un an auparavant, il avait sondé la poésie de Hölderlin, en tâtonnant déjà cette pure langue, pensée encore, en 1915, comme une sorte de noyau poétique (das Gedichtete). Justement, dans la co-relation de deux poèmes, deux versions en deux langues presque différentes: écrits par Hölderlin, dans sa maturité d'une part (Dichtermut, Hölderlin 1801/2003) ${ }^{13}$, dans sa dernière période d'autre part (Blödigkeit, Hölderlin 1801/2003 $)^{14}$. Il n'est pas surprenant que le jeune Benjamin ait lu ces deux poèmes presque comme un traducteur, non pour les traduire vers une autre langue - comme le fera Riba dans ses Versions de Hölderlin (1943-1944) ${ }^{15}$-, mais pour racheter ce noyau poétique, exilé à la fois dans les deux poèmes hölderliniens.

Très tôt, pressé pour un manque de revenus qui avec le temps deviendra chronique, convaincu de la nécessité d'une étape d'invisibilité dans les relations entre Juifs et Allemands, Benjamin commencera à voir dans le français et l'hébreu une possible patrie d'élection (Wahlheimat). Sa traduction des Tableaux parisiens de Baudelaire $(1923 / 1972)^{16}$, mais surtout ses réflexions autour de la tâche du traducteur, font comprendre que Benjamin pensait tout aspect se référant à l'idée d'ethnie sous forme linguistique, comme il l'explique à son ami Christian Rang, à la fin de 1923: «Il me serait essentiel de parler un jour avec toi de la question des ethné. Elle ne m'est apparue jusqu'ici que sous l'aspect du langage, ainsi que j'ai commencé à m'en expliquer dans l'introduction à la traduction de Baudelaire» (Benjamin 1979: 291, traduction de Guy Petitdemange). Une introduction dans laquelle le texte biblique et 
les traductions de Hölderlin, surtout celles des deux tragédies de Sophocle (Antigone et $(E \text { dipe })^{17}$ fonctionnent non seulement comme modèles de la tâche du traducteur, mais surtout comme preuves de l'existence de cette pure langue exilée dans chaque langue.

Tandis que Riba, en tant que traducteur, fait passer au catalan cette pure langue, en tant que poète, il fera passer sa propre voix à travers la voix de Hölderlin. Ainsi, surtout dans les Elegies de Bierville, où Riba essaiera de traduire «O Gesang den Reinen», exclamation non traduite dans sa version incomplète de An die Madonna (Hölderlin 1805/1923 : 254; Riba 1943-1944: 37) ${ }^{18}$, mais qu'on peut lire, en revanche, dans la sixième Elegie de Bierville: «l'incalculable mot, pur en l'espera dels déus! » (le mot incalculable, pur dans l'attente des dieux! Riba 1942: 223, traduction de l'auteur $\left.{ }^{19}\right)$.

Si Hölderlin avait déjà attendu ce mot incalculable dans la foi en la distance entre lui et les dieux, c'est probablement parce que cette distance avait converti pour lui déjà sa propre langue - l'allemand de 1800 - en une langue à la fois maternelle et étrangère. Dans son carnet de notes, Riba écrit:

En el fet poètic (en la inspiració), la pròpia llengua esdevé per al poeta una llengua estrangera que el condueix més que no pas la condueix ell [...]. (Riba 1988:335)

Dans le fait poétique (dans l'inspiration), la langue propre devient elle-même pour le poète une langue étrangère, qui le conduit plus qu'il ne la conduit [...]. (Traduction de l'auteur)

C’est pour cela qu'il faut écouter les derniers hymnes de Hölderlin d'une oreille grecque puis d'une oreille haut-allemande, c'est-à-dire, avec la distance co-relative entre la langue de Sophocle et la langue de Luther. Selon Berman,

[p]ar le dialogue avec le grec et le retour à l'élément dialectal de l’allemand, la poésie [et la traduction (ajout de l'auteur)] f[ont] accéder la langue commune à sa dimension propre, à cette dimension d'équilibre entre la langue étrangère et le dialecte qui est son origine. (Berman 1984/2007: 266)

Une lecture de Hölderlin et une vision de la traduction que Berman élargira jusqu'à l'horizon traductologique de Benjamin, comme en témoigne son commentaire sur «La tâche du traducteur» (Berman 2008), texte lu par Berman comme une sorte de révélation du lien entre cette pure langue et le dialecte, en tant que dépôt - presque géologique - de la tradition, celle-ci entendue comme oralité.

Cette corrélation, Riba, de retour d'exil, essaiera de la rétablir pour remonter le mythe homérique aux sources orales de sa llengua viva. On peut lire ainsi sa deuxième version de l'Odyssée - libérée seulement en partie de l'hexamètre homérique - comme un plongeon dans une rivière lointaine, parsemée de pierres natales (colloquialismes, dialectalismes, ultra-corrections populaires). Quand Ulysse - de retour à Ithaque - est traité de «mendiant» par le chevrier Mélanthée, Riba n’hésitera pas à traduire ce substantif grec $(\pi \tau \omega \chi \grave{o} v \text {, [ptōchon] })^{20}$ par un emprunt castillan (gallofa ou gallofero, 'fainéant') au latin médiéval (galli offa, 'bouchée du pèlerin'), ultra-correction populaire de ce dialectalisme médiéval, catalanisé par une tendance à ajouter un $-l$ parasite aux mots terminés par - $o$ atone, ressentis comme castillans (Bruguera 2001: 361). 
¿On duus aquest golafràs, porquerol qui a ningú fas enveja, aquest gallòfol ronser, aquest empastifa-dinades?

(Homère VIII s. av. J.-C./1953: 304, traduit par Riba; souligné par l'auteur)

Misérable porcher, où conduis-tu donc ce glouton, ce mendiant importun, ce fléau des festins?

(Homère VIII' s. av. J.-C./2004: 280, traduit par Jaccottet ${ }^{21}$; souligné par l'auteur)

Traduction qu'on peut écouter - du moins, en interlignes - comme une sorte d'hommage secret à la mémoire d'Antonio Machado, en souvenir des paroles que le poète castillan lui avait adressées, juste après avoir passé la frontière franco-espagnole, le 28 janvier 1939: « ¡Hallarme en Francia, y como un mendigo!» (Me trouver en France, comme un mendiant!).

Si le $\mathrm{xx}^{\mathrm{e}}$ siècle fut celui de la traduction, on peut dire sans aucun doute qu'il fut aussi celui de l'exil. Il n'est pas rare que Benjamin et Riba - qui ont toujours compris la traduction dans la dimension de la vie - y voient une métaphore de l'exil. Exil d'une langue utopique, mais à la fois réelle, une langue perdue et pourtant une langue-qui-vient. Une langue pure et vive - adjectifs d'évidente ascendance hölderlinienne - qu'on parlera un jour, mais qu'on parle toujours chaque fois qu'on traduit un texte en dehors de la langue d'origine et de la langue d'accueil.

\section{NOTES}

1. Carles Riba. Passió i lucidesa (1993): Réalisé par Josep Oller. Barcelone, Producció Iniciatives Àudio-Visuals.

2. Virgile (-39 av. J.-C. /1911): Les Bucòliques de Virgili. (Traduit par Carles RiBA). Barcelone: D. F. Altés Alabart.

3. Homère (viII e s. av. J.-C./ 1919): Odissea. (Traduit par Carles RibA). Barcelone: Editorial Catalana.

4. Malgré le fait d'avoir coïncidé à Berlin pendant la fin de l'été de 1922, avant que Benjamin ne parte à Francfort, et Riba à Munich (Benjamin 1979: 266; Riba 1989: 212).

5. Riba fera ainsi appel à une terminologie plus linguistique que métaphysique, afin d'essayer d'arriver à cet impossible qui est de remplacer l'irremplaçable (substituir l'insustituïble), de faire du contenu une forme (contingut una forma) (Riba 1987: 124), presque de la même façon que Benjamin voulait faire du symbolisant le symbolisé (das Symbolisierende zum Symbolisierten selbst zu machen) (Benjamin 1974: 19).

6. Sous l'épigraphe de Literatura expressionista, Riba va traduire Der Mord (sous le titre de Un fratricidi), un petit conte écrit par Kafka en 1917, qui est, en fait, la première version d'Ein Brudermord, texte recueilli plus tard dans Kleine Erzählungen, par Ein Landarzt. LANDARzT, Ein (1920): Kleine Erzählungen. Munich/Leipzig: Kurt Wolff.

7. Homère (viII ${ }^{\mathrm{e}}$ s. av. J.-C./1948) : L'Odissea. (Traduit par Carles RiBA). Barcelone: Societat Aliança d'Arts Gràfiques. La deuxième édition de cette deuxième traduction est parue en 1953: HomèrE (viII ${ }^{\mathrm{e}}$ s. av. J.-C./1953) : L'Odissea. (Traduit par Carles RibA). Barcelone: Editorial Alpha. Une autre traduction est celle de Fernando Gutiérrez. Homère (viII ${ }^{e}$ s. av. J.-C./1951) : Odissea. (Traduit par Fernando GutiérREZ). Barcelone: José Janes Editor.

8. Carles Riba (1919): El darrer freu, Estances. Barcelone: La Revista. Carles Riba (1942): Elegie VII, Elegies de Bierville. Buenos Aires/Barcelone: sans maison d'édition. Ces poèmes ont été écrits en 1919 pour les premiers, et 1940, pour les seconds.

9. Carles Riba, dans Elogi del poeta traductor, écrit: « [...] veure i apprendre, això es, segons l'eterna fórmula que resumí els viatges d'Ulisses» (apprendre et voir, cela se fait selon la formule éternelle résumée dans les voyages d'Ulysse; Riba 1985: 85, traduction de l'auteur).

10. Le poème date de 1948. Riba, Carles (1984): Obres completes. Poesia. In: Enric Sullà, dir. Vol. 1. Barcelone: Edicions 62.

11. Dans le chapitre: «Hölderlin: le national et l'étranger». 
12. HöLDERLIN, Friedrich (1801/2003): Heimkunft. Stutggart: Reclam.

13. Hölderlin, Friedrich (1801/2003): Dichtermut. Stutggart: Reclam.

14. HöLDERLIN, Friedrich (1801/2003): Blödigkeit. Stutggart: Reclam.

15. Riba, Carles (1943-1944): Versions de Hölderlin. Buenos Aires/Barcelone: sans maison d'édition.

16. Baudelaire, Charles (1857/1972): Tableaux parisiens. Deutsche Übertragung mit einem Vorwort über die Aufgabe des Übersetzers von Walter Benjamin. (Traduit par Walter Benjamin 1923). Heidelberg: Verlag von Richard Weissbach.

17. Sophocle (ve s. av. J.-C./1804/1982): Antigone. Öedipus (Traduit par Friedrich Hölderlin). Harenberg Verlag: Dortmund.

18. HöLderlin, Friedrich (1805/1923): An die Madonna. Berlin: Propyläen Verlag.

19. Riвa, Carles (1942): Les Elegies de Bierville. Buenos Aires/Barcelone: sans maison d'édition.

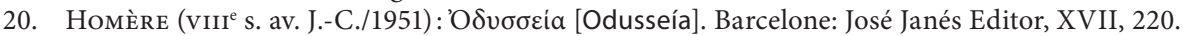

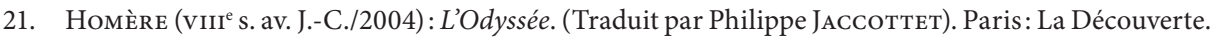

\section{RÉFÉRENCES}

Benjamin, Walter (1972): Die Aufgabe des Übersetzers. In: Rolf Tiedemann, dir. Gesammelte Schriften. Frankfurt am Main: Suhrkamp, 9-21.

Benjamin, Walter (1977): Franz Kafka. In: Rolf Tiedemann, dir. Gesammelte Schriften. Frankfurt am Main: Suhrkamp, 409-438.

Benjamin, Walter (1979): Correspondance. 1910-1928. (Traduit par Guy Petitdemange) Paris: Aubier.

Benjamin, Walter (1993): Briefe. Frankfurt am Main: Suhrkamp.

Berman, Antoine (1984/2007): L'épreuve de l'étranger. Paris: Gallimard.

Berman, Antoine (2008): L'âge de la traduction. "La tâche du traducteur» de Walter Benjamin, un commentaire. Paris: Presses universitaires de Vincennes.

Bruguer a, Jordi (2001): Sufixos fòssils del català. In: Jopep Massot i Muntaner, dir. Estudios de llengua i literatura catalanes/XLII. Miscelllània Giuseppe Tavani. Barcelone: Publicacions de l'Abadia de Montserrat, 333-372.

Ferrater, Gabriel (1979): Sobre literatura. Barcelone: Edicions 62.

MalÉ, Jordi (2006): Carles Riba i la traducció. Barcelone: Punctum \& Trilcat.

Medina, Jaume (1987): Carles Riba i Friedrich Hölderlin. Barcelone: Publications de l'Abadia de Montserrat.

RANCIÈRE, Jacques (1998): La parole muette. Essai sur les contradictions de la littérature. Paris: Hachette.

RibA, Carles (1953): Uns mots del traductor. In: Homère (viII ${ }^{\mathrm{e}}$ s. av. J.-C./1953): L'Odissea. (Traduit par Carles RiBA). Barcelone: Editorial Alpha, 7-15.

RiBA, Carles (1985): Obres completes. Crítica 1. In: Enric SulLÀ, dir. Vol. 2. Barcelone: Edicions 62.

RibA, Carles (1986): Obres completes. Crítica 2. In: Enric Sullà et Jaume Medina, dir. Vol. 3. Barcelone: Edicions 62.

Riba, Carles (1987): Clàssics i modernes. In: Joaquim Molas, dir. Barcelone: Edicions 62.

Riba, Carles (1988): Obres completes. Crítica 3. In: Enric Sullà et Jaume Medina, dir. Vol. 4. Barcelone: Edicions 62.

Riba, Carles (1989): Cartes de Carles Riba. 1910-1938. In: Carles-Jordi Guardiola, dir. Vol. 1. Barcelone: Institut d'Estudis Catalans.

Scholem, Gershom (1926/1985): Une lettre inédite de Gershom Scholem à Franz Rosenzweig. À propos de notre langue. Une confession. (Traduit par Stéphane Mosès). Archives de sciences sociales des religions. 60(1):83-84.

Scholem, Gershom (1970/2006): El nombre de Dios y la teoría del lenguaje de la cábala. (Traduit par José Luis BARbero SAMPEDro) In: Gershom Scholem, dir. Lenguajes y cábala. Madrid: Siruela, 11-100. 